intermediate stratum of air that we must ascribe (exempli gratia) the doubt whether Kinchinjunga or $\mathrm{K}_{2}$ is to hold the honourable position of second in altitude to Everest amongst the world's highest peaks.

T. H. H.

\section{REPORTS OF MUSEUMS.}

THE report of the Bristol Museum for the year ending September 30, I9I3, records praiseworthy activity, especially in the department of vertebrate zoology. Three plates show how attractively some of the more important specimens are displayed. A tiger shot by the King in Nepal, and presented by his Majesty, has been set up by Messrs. Rowland Ward, in a crouching attitude among bamboo stems, while the background, painted by Mr. Stanley Lloyd, shows the shooting-party approaching on elephants in the distance. Three springboks are placed near the margin of a veldt, on which other animals are brows ing; this background was painted by Mr. G. E. Butler. The picturesque group in which pheasants are feeding (harmlessly) in the stubble, is backed by a view of Ashton Park, with the Clifton Suspension Bridge in the distance, composed by Mr. A. Wilde Parsons. This utilisation of really competent artists is an example to be followed. The geological department has not shared in the general progress, and considering the recent work of Vaughan and others in the west of England, this fact is rightly deplored by the committee.

With the aid of local naturalists, the small staff of the Hancock Museum at Newcastle-upon-Tyne has during the past two years accomplished some excellent work. From a $45-\mathrm{ft}$. Rorqual (Balaenoptera borealis) cast ashore near Amble, the complete skeleton, including ear-bones, hyoids, and rudimentary hip-bones, was obtained. The larger bones have been satisfactorily prepared in a sand-pit; but the smaller bones which were macerated as usual in water made so little progress that they have now been transferred to sand. A promising beginning was made with classes from the elementary schools, each of which went through a definite course of six lessons, given by the teachers, who were first rehearsed in the lesson by the curator, Mr. E. L. Gill. Unfortunately this regular system could not be followed in the second year, owing to the overcrowded curriculum of the schools, and the visits are now of small educational value. Perhaps the committee recently appointed by the British Association may devise some scheme that will overcome this difficulty.

The report of the Manchester Museum for the year I9I2-I 3 bears witness to plenty of hard work, but contains nothing of outstanding interest. It is, however, worth reading in order that one may admire the healthy spirit of cooperation as regards museum matters that breathes in Manchester. Representatives of the University, of the City Council, and of subscribers among the outer public, constitute the committe of management. The City Council has increased its grant from 40ol. to $800 l$. per annum. Professors of the University supervise and aid the museum staff. In the transference of the Egyptian antiquities to the new building, which, with its cases, was provided by Mr. Jesse Haworth, valuable help was given by a number of ladies and gentlemen. Several ladies have maintained a supply of fresh flowers, and at least four other names are mentioned in connection with solid pieces of work of more expert character. To a museum combining so many forces there naturally flow considerable donations, both in money and in kind.

$$
\text { NO. } 2315 \text {, VOL. 93] }
$$

\section{RADIATION OF GAS MOLECULES EXCITED BY LIGHT.}

THE first Guthrie lecture of the Physical Society was delivered on February 27, at the Imperial College of Science, South Kensington, by Prof. R. W. Wood, of Johns Hopkins University, Baltimore. The lecture has been established in memory of Prof. F. Guthrie, who was professor of physics in the Royal College of Science, and was founder of the society, the first meeting having been held in his lecture theatre at the college in 1874 . Before Prof. Wood's lecture, Prof. G. Carey Foster gave a short biography of Guthrie, who was born in 1833 and died in 1886, and Sir Oliver Lodge recalled some personal reminiscences of him. Prof. Wood's lecture is summarised in the subjoined abstract, published by the Physical Society.

The emission and absorption of light by molecules and the allied phenomenon of dispersion have led us to the conception of something within the atom which is capable of responding to light waves in much the same way as a tuning-fork responds to sound waves of the same frequency as its own, and many mathematical treatments have been built up which explain more or less perfectly many of the phenomena in question. These still leave us very much in the dark as to what is going on. Helmholtz explained absorption by introducing a frictional term into his equations of motion for the atom, and though this led at once to an expression which represented anomalous dispersion, it left us ignorant of how the energy absorbed by the molecules was transformed to heat, or how the mean velocity of the molecules was increased by the excitation of vibrations within them. Planck avoided this difficulty by considering that the energy abstracted from the beam of light is re-emitted, though at the time the only experimental evidence was to be found in selective reflection, which occurs only in liquids and solids.

What becomes of the absorbed energy in the case of a gas? This was what he had been asking himself for many years. While he did not require a working model of the atom, he could not, however, be satisfied by an equation in which absorption was represented by a frictional term or selective reflection predicted by the occurrence of an imaginary quantity.

The problem of the constitution of the atom is one which must be approached from many sides, as it is improbable that any single mode of attack will reveal the secret. The spectroscope alone has proved itself powerless, one great difficulty being that in all known methods of cxciting spectra one got "the whole or nothing."

Flames, arcs, sparks, and vacuum-tube discharges set a host of vibrations simultaneously in operation within the atom, and resulted in a complex of lines which were difficult to interpret.

His line of attack had been to maintain the molecules in as calm and tranquil a state as possible, by keeping them cool, and then to excite them to radiation by the application of an alternating electromagnetic field of a definite frequency-usually called monochromatic light. That this method has in some degree simplified matters was proved by the fact that sodium vapour could be made to emit only one of the D lines instead of the usual two.

The conditions necessary to stimulate radiation in this way varied considerably with the nature of the element studied. He would begin, however, with the simplest case, that of a vapour which exhibits a single absorption line and emits radiations similar in every respect to the exciting radiations when stimulated by 
light of frequency equal to that of the absorption line. This condition was perfectly fulfilled by the vapour of mercury, which has an absorption line at $\lambda=2536$ in the ultra-violet.

If a beam of monochromatic light of this wavelength was focussed at the centre of an exhausted quartz bulb containing a drop of mercury at atmospheric temperature, it was found that the light was powerfully scattered by the vapour, photographs of the bulb made with a quartz lens showing the cone of rays much as if the bulb were filled with smoke. The scattered light is invariably much more homogeneous than the incident beam, in which the "line" has a finite width, whereas the scattered light corresponds only with the centre of this line. "The rest gets through the vapour unaffected. With the light thus scattered-the resonance radiation-a photograph was made of a quartz bulb containing a minute drop of mercury at room temperature. The bulb appeared as if filled with ink owing to the opacity of the vapour for the rays.

These phenomena, visible only to the camera, can be visually reproduced in the case of sodium vapour excited by the light from a sodium flame. If the density of the vapour is increased by warming it, the distance which the light can penetrate into the bulb is diminished and eventually the resonance radiation is all emitted from a region so close to the surface that it appears as a bright yellow patch on the inner surface of the glass.

If this patch is now used as a lamp, and focussed by a concave mirror on the surface of the same globe (or another in which the vapour is of sufficient density to give the patch effect) so as to fall partly on a surface whitened by deposited magnesia and partly on the enclosed vapour, the brightness of the two contiguous patches thus formed is practically equal.

This proves that, under those conditions, at comparatively low densities, true absorption does not exist, the light abstracted from the incident beam being reemitted as light of the same wave-length but in all directions.

The factor of true absorption makes itself manifest as soon as we admit air or some other foreign gas. Even if the pressure is only a millimetre or two the effect is very marked.

Another point which can be brought out by this method of attack is whether or not the mechanisms the vibration frequencies of which correspond to the various lines in a spectrum are independent of each other or are interconnected.

An ingenious method was described whereby a beam of considerable intensity, consisting, however, of only $D_{1}$ or $D_{2}$ light, could be obtained, and if the sodium vapour excited by either of these was examined spectroscopically the emitted light contained only that one of the lines which was used to excite it. This shows that the $D_{1}$ and $D_{2}$ mechanisms are quite independent. In other cases, however, vapours excited by light of any one line of their spectrum gave out a resonance spectrum of that line and one or more others showing that some groups of mechanisms were interdependent and could not be excited separately.

Stimulation by Waves of Very Short Wave-Length. -Experiments were then described in which air, nitrogen, etc., had been caused to emit ultra-violet light when exposed to the action of radiation of wavelength less than the Schumann rays, the smallest waves hitherto known. Schumann rays were completely absorbed by quartz, but would pass through a considerable thickness of fluorite, but the rays to which he referred could be reduced in intensity by 98 per cent. by a plate of fluorite $1 \mathrm{~mm}$. thick.

Nitrogen was more actively stimulated than air by these rays, as oxygen seemed to have a destructive effect on the phenomena. Thus iodine vapour, if mixed with nitrogen, emitted a green light under the action of the rays, while remaining dark if mixed with oxygen.

He urged the necessity of an exact mathematical treatment of the phenomenon of a molecule of vapour re-enitting radiation which it has abstracted from an incident beam, truc absorption being absent.

At the conclusion of the lecture a number of interesting experiments illustrative of the subject of the lecture were shown. These included the resonance radiation of sodium stimulated by $D$ light, of iodine vapour stimulated by the light from a quartz-mercury lamp, and of the author's method of extinguishing one of the $\mathrm{D}$ lines from the light from a sodium flame.

\section{STRUCTURAL ANALOGIES BETWEEN IGNEOUS ROCKS AND METALS.}

I T was in Sheffield that the late Dr. H. C. Sorby lived and worked. It was to the Sheffield Literary and Philosophical Society that, in 1864 , Sorby presented the first account of his microscopical examination of the structures of commercial steel. In Sheffield the worth of Sorby's work is now being recognised, and during the presidency of Mr. Arthur Balfour the Sheffield Society of Engineers and Metallurgists, an active and growing society closely associated with the industries of the city, has founded the "Sorby Lecture," to "mark its progress," and to perpetuate the memory of its late honorary member.

The first Sorby Lecture, on February 28, was the occasion for a large gathering of Sheffield's leading manufacturers and citizens at the Cutlers' Hall. The lecture was delivered by Prof. W. G. Fearnsides, the occupant of the Sorby chair of geology at Sheffield University, "On Some Structural Analogies between Igneous Rocks and Metals."

In the first part of the lecture Prof. Fearnsides traced the progressive development of the research by which Sorby, already trained to a knowledge of optics and of chemistry, learned from Williamson the art of making transparent sections of hard objects, and applied it (1849) to the study of rocks. Limestones were the first rocks to claim his attention (185I), then slates ( 1856 ), and then igneous rocks (1857), and from these, through meteorites (1862), he was led to study irons $\left(1863_{-4}\right)$. The difficulties which Sorby encountered and his patient toil, continued in defiance of indifference and ridicule, were discussed, and it was conjectured that the apathy with which his results were received was due to his own inability to appreciate the difficulties which his refined technique and the vector variations of the optical properties of minerals presented to other people.

The recognition of the value of Sorby's petrographic methods grew gradually through the sixties and seventies of last century, but it was not until after his announcement to the Iron and Steel Institute in I886, that in the previous year a new microscope had enabled him to see the true composite nature of the "pearly constituent" of steel, that his pioneer work on metals attracted any attention.

It was by a fortunate but unforeseen coincidence that the first Sorby Lecture was delivered within a few days of the fiftieth anniversary of the day on which Sorby read the first of all papers dealing with the micro-structure of commercial metals, and the subject for the lecture was chosen accordingly.

The second part of the lecture dealt with the modern view that igneous rocks and metals are alike products derived by progressive partition of components during the crystallisation of mixed solutions. Being thus

NO. 23I5, VOL. 93] 